\title{
Regularized InfraRed Image Correlation Applied to Laser-Induced Thermal Shocks
}

\author{
by A. Charbal*,**, S. Roux*, F. Hild* and L. Vincent** \\ *LMT Cachan, ENS-Cachan / CNRS / Univ. Paris-Saclay, 61 av. du Président Wilson, F-94235 Cachan cedex, \\ France, \{charbal,stephane.roux, hild\}@Imt.ens-cachan.fr \\ ${ }^{* *}$ DEN, Service de Recherches Métallurgiques Appliquées (SRMA), CEA, Université Paris Saclay, F-91191 Gif \\ sur Yvette cedex, France, ludovic.vincent@cea.fr
}

\begin{abstract}
The thermomechanical behavior of a stainless steel sample undergoing thermal shocks is investigated with an infrared (IR) camera. The temperature and kinematic fields are simultaneously determined exploiting only IR frames. The thermal loading is prescribed with a laser, which gives rise to very high local digital level variations. This effect is addressed thanks to a regularized infrared image correlation (R-IRIC) technique, which accounts for brightness and contrast variations. The resulting digital level residuals are reduced down to less than $1 \%$ of the dynamic range, i.e. a level comparable with the noise magnitude, thereby validating the approach.
\end{abstract}

Keywords: DIC, Digital levels, Regularized-IRIC, Thermomechanical fields

\section{Introduction}

The use of IR cameras in the field of experimental mechanics is more and more common [1-13] in order to quantify the thermomechanical response of materials under load. Most of the time the sought information consists of the kinematic and temperature fields. Both are induced by either the mechanical loading [1-9,11-12] or the applied thermal history [10,13-14]. In the first case, temperature elevations are of few degrees Celsius, and accessing the thermomechanical response may provide a better understanding of localization and dissipative phenomena [1-9,11-12]. In the second case, the temperature variation can reach few hundred degrees and induces local deformation of the sample [10,13-14]. In both cases, the measurements of temperature and displacement fields are of interest. The IR frames, after suitable calibration procedures, provide the temperature fields. The use of digital image correlation (DIC), which is performed on images shot during the test usually with a camera working in the visible light domain, can reveal the kinematic fields. The IR frames may also be used to reach such information but the digital level ${ }^{1}(\mathrm{DL})$ variations that occur due to temperature variations may be an issue, as most DIC algorithms are based upon the DL conservation assumption. Another problem related to the surface state (emissivity) should be dealt with as a contrasted pattern is necessary to perform DIC. However additional calibration steps may overcome such problem. For instance a black body in reflection to the IR camera can be used, or the sample may be uniformly heated at a controlled temperature and the emissivity field may be estimated.

Some experimental strategies have been proposed to have access to such experimental quantities. One of them requires the use of two cameras, namely, one working in the IR and the second in the visible light wavelength range [1$9,10,13-14]$. The visible light camera is not sensitive to temperature variations and the DL conservation assumption is satisfied. The DIC algorithms can be used and by means of synchronization, the IR frames (with temperature information) and 2D DIC displacement fields can be related. The difficulties are that the cameras should be positioned close to each other to image the same regions of interest. The IR frames may be affected by the environment if small temperature variations are to be captured. Too large an angle of view may induce (parallax) distortions. The use of a dichroic mirror, which permits one camera to be parallel to the sample surface and the second one at $90^{\circ}$ has shown to be a satisfactory alternative [6]. Calibrations are then necessary to relate the zone of temperature variations to displacement fields [8]. The use of thin samples, which allow the two cameras to be positioned on each side, has also been proposed [4-5]. A less constraining method consists in integrating in the DIC formalism (referred to as InfraRed Image Correlation or IRIC) the occurrence of these DL variations and then use the IR frames. The feasibility of such a method was shown for a single IR camera and temperature variations of few degrees [12]. The DIC formalism consists in considering a sort of "brightness" correction decomposed onto a Finite Element (FE) basis. Some additional experimental steps were performed to capture the emissivity field and provide at convergence the 2D displacement and the (Lagrangian) temperature fields.

In this work, it is proposed to use a generalized DL correction by considering the effect of temperature variation (on the shot IR frames) as being contrast and brightness changes [15]. These variations can be added to a global DIC framework [15] and those two fields can also be decomposed onto an FE basis as for displacement fields [16]. A mechanical regularization [17] can be applied to filter out inherent fluctuations due to noise. In addition to the latter, it is proposed herein to apply regularization to the $\mathrm{DL}$ fields. The filter is a penalty given to steep temperature gradients. The

1 In this paper the exploited IR frames are converted into temperature $\left({ }^{\circ} \mathrm{C}\right)$, the use of "DL" instead of "temperature" is just to be consistent with the terminology used in image processing. Some quantities will be later discussed such as contrast and brightness fields for which the term "DL" is more appropriate and should avoid confusion with temperature field. 


\subsection{1/qirt.2016.019}

main goal of this study is to measure kinematic fields with IR frames, the temperature information being already within the exploited images for IRIC calculations. The necessary steps for thermography measurements are already discussed in Ref. [14] and the same ones are applied herein (to determine the averaged surface emissivity).

The remainder of paper is divided into three parts. The motivations are detailed in Section 2 considering the effect of temperature variations on the DL distributions. The R-IRIC formalism is detailed in Section 3 while the results are discussed in Section 4.

\section{Motivations}

The thermomechanical behavior of $316 \mathrm{~L}(\mathrm{~N})$ stainless steel subjected to thermal shocks is investigated with an IR camera (figure 1). The characterized sample is a polished $70 \times 40 \times 7 \mathrm{~mm}^{3}$ parallelepiped. In order to measure displacement fields by image correlation, a random pattern (e.g. pre-oxidation or laser engraving [13]) is applied onto an initially polished surface of the sample. A pulsed laser (TruPulse 156 manufactured by Trumpf, $\lambda=1064 \mathrm{~nm}$ ) is used to induce cyclic thermal shocks in the center of one of the two largest faces of the samples, starting from a base temperature of $400^{\circ} \mathrm{C}$ [18]. A focusing optics delivers a top-hat power density whose diameter is $5 \mathrm{~mm}$. Due to a relatively low absorptivity of the polished surface, the incident beam is inclined in order for the reflected beam to be collected by a calorimeter. An infrared camera (x6540sc manufactured by FLIR, definition: $640 \times 512$ pixels, $\lambda=3970-4010 \mathrm{~nm}$ ) with a high magnification lens is used to measure simultaneously the displacement and temperature fields [12].

(a)

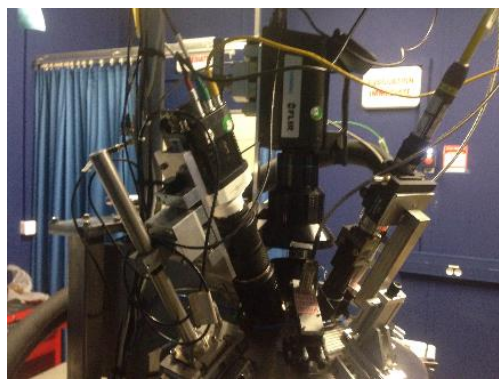

(b)

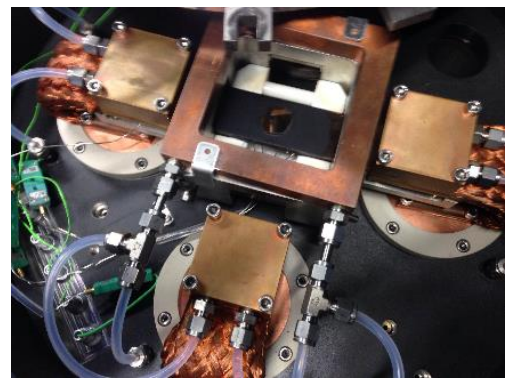

Fig 1: (a) Experimental bench and its IR camera with G1 (center) lens and the laser source (right). (b) Stainless steel sample onto which the thermal shocks are applied

The main assumption of DIC is the DL conservation between the registered images. The optical flow is then measured once the displacement fields are properly estimated. For the use of IR frames with anisothermal sequences, where DLs vary with temperature, the assumption is no longer fulfilled. When laser shocks are applied, the IR frames are highly and locally disturbed (for DIC purposes), see figure 2.

(a)

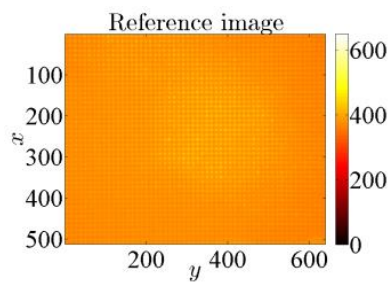

(b)

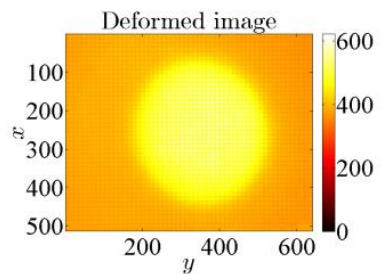

(c)

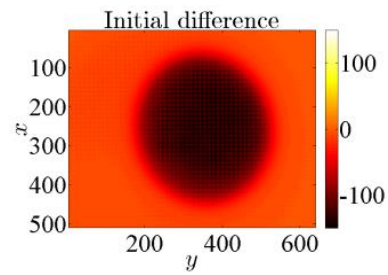

Fig 2: Reference (a) and deformed (b) IR images.

(c) Initial correlation residuals (RMS: $54 \%$ of the dynamic range)

An illustration on a row of pixels is proposed in order to show the effect on the compared signals. Figure 3(a) shows the effect on the DL profile before and during temperature elevation. An affine brightness and contrast correction seems to recover most of the DL variations (figure $3(b)$ ) when the coefficients of the correction are allowed to vary spatially, yet more slowly than the original image texture. 


\subsection{1/qirt.2016.019}

(a)

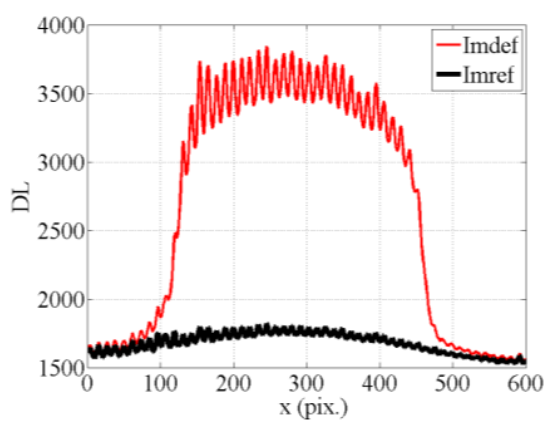

(b)

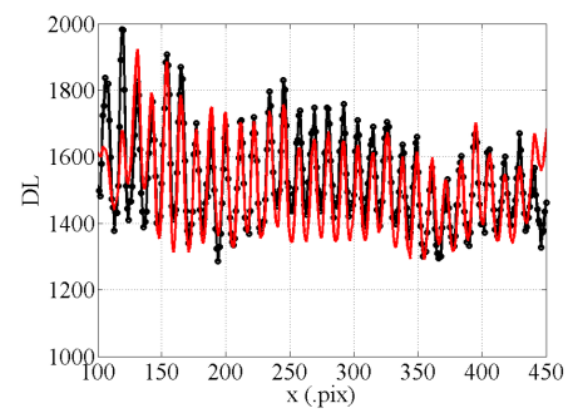

Fig 3: Digital levels on one row of pixels taken on the deformed (red) and reference (black) pictures (a) before any correction, and (b) with brightness and contrast corrections $(f=a+(1+b) g)$

This simple analysis on a row of pixels suggests that DL corrections can be incorporated in a global DIC formulation, over a region of interest $(\mathrm{ROI})[15]$

$$
\Phi_{c}^{2}=\sum_{R O I}\left(f(\boldsymbol{x})-a(\boldsymbol{x})-(1+b(\boldsymbol{x})) g_{0}(\boldsymbol{x}+\boldsymbol{u}(\boldsymbol{x}))\right)^{2}
$$

where $f$ and $g_{0}$ correspond to the reference and deformed images. The brightness $(a)$, contrast $(b)$ and displacement vector $(\boldsymbol{u})$ fields are decomposed onto an FE basis

$$
\begin{aligned}
& a(\boldsymbol{x})=\sum_{k=1}^{P} a_{k} \psi_{k}(\boldsymbol{x}) \\
& b(\boldsymbol{x})=\sum_{k=1}^{P} b_{k} \psi_{k}(\boldsymbol{x}) \\
& \boldsymbol{u}(\boldsymbol{x})=\sum_{k=1}^{P} u_{k} \boldsymbol{\psi}_{k}(\boldsymbol{x})
\end{aligned}
$$

A Newton-Raphson algorithm based on successive linearizations is used to solve iteratively the minimization of $\Phi_{c}^{2}$. The linear system (to be recomputed at each step $n$ ) reads

$$
\left[M^{n}\right]\left\{\delta U^{T}\right\}=\left\{b^{n}\right\}
$$

or

$$
\left[\sum_{R O I}\left(\left(m^{n T} \otimes m^{n}\right)(x)\right)\right]\left\{\delta U^{T}\right\}=\left\{\sum_{R O I}\left(m^{n T}(x)\left(f-g^{n}\right)(x)\right)\right\}
$$

where all unknown amplitudes are gathered in a single vector $\{\boldsymbol{\delta} \boldsymbol{U}\}$

$$
\{\boldsymbol{\delta} \boldsymbol{U}\}^{\boldsymbol{T}}=\{\delta a, \delta b, \delta \boldsymbol{u}\},
$$

and the vector $\boldsymbol{m}^{\boldsymbol{n}}$ reads

$$
\boldsymbol{m}^{\boldsymbol{n}^{T}}(\boldsymbol{x})=\left\{\psi, \psi g_{0}^{n}, \boldsymbol{\psi} \cdot \boldsymbol{\nabla} \boldsymbol{f}\right\}(\boldsymbol{x})
$$

the deformed image is corrected by the current determination of the displacement and DL fields

$$
g^{n}(\boldsymbol{x})=a^{n}(\boldsymbol{x})+\left(1+b^{n}(\boldsymbol{x})\right) g_{0}^{n}(\boldsymbol{x}) \text { and } g_{0}^{n}(\boldsymbol{x})=g_{0}\left(\boldsymbol{x}+\boldsymbol{u}^{n}(\boldsymbol{x})\right)
$$

The $\left[\boldsymbol{M}^{\boldsymbol{n}}\right]$ matrix is approximated as blocks of matrices because the coupling terms between DL and displacement fields are small enough to be neglected

$$
\left[\boldsymbol{M}^{\boldsymbol{n}}\right]=\left[\begin{array}{cc}
\boldsymbol{M}^{\boldsymbol{n}}{ }_{D L} & \mathbf{0} \\
\mathbf{0} & \boldsymbol{M}_{u}
\end{array}\right]
$$




\subsection{1/qirt.2016.019}

$\boldsymbol{M}_{D L}^{\boldsymbol{n}}$ contains the products of the DL correction fields and $\boldsymbol{M}_{u}$ contains the dyadic product of the image gradient $f$ all multiplied by the shape functions. The iterative algorithm is driven up to the stage where the incremental changes in $\mathrm{DL}$ correction and displacement parameters become less than a chosen threshold $\left(10^{-5}\right.$ in the present case).

The mesh size should be chosen depending on the pattern quality and DIC resolution to be reached [19]. Here because sharp gradients are to be captured, the selected mesh size is 12 pixels (figure 4). The discretization enables for the assumption within an element that linear DL corrections are a good approximation. The elements near the edges (of the laser spot) may suffer from such an assumption and give rise to larger residuals (this effect will be illustrated later on).

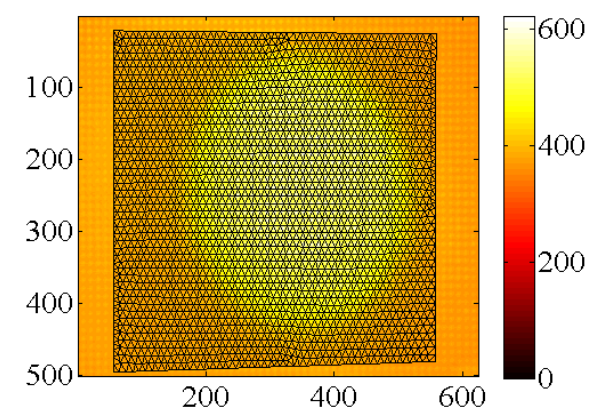

Fig 4: FE mesh for global DIC analyses

The contrast and brightness corrections introduced in the global "FE-DIC" framework allow kinematic fields (equivalent plots are given in Section 4) to be well captured and yield residuals below $1 \%$ of the dynamic range. It can be noticed that the DL fields are "noisy" (figure 5). If smaller element sizes are considered (e.g. 8 pixels), say, to reach a better spatial resolution (for displacement fields), the fluctuations observed on the brightness and contrast fields limit the convergence of the IRIC algorithm. The fluctuations are not due to the temperature (DL) distribution but are a manifestation of the ill-posedness of the problem. One way to circumvent such difficulties is to apply a regularization to guide the solution as will be discussed in the following section.

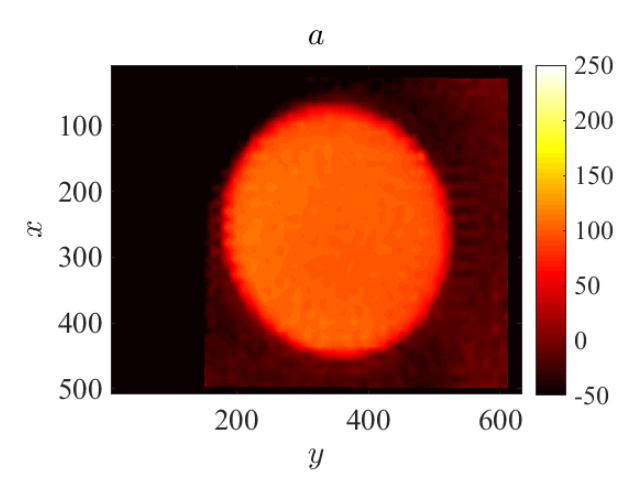

(a)

Fig 5: Brightness

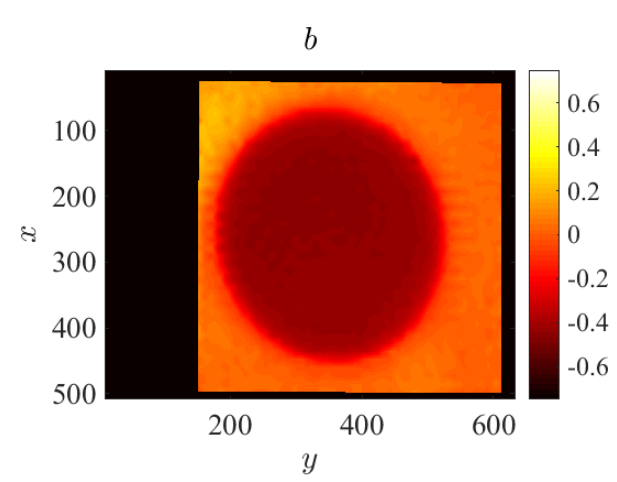

(b)

\section{Regularized IRIC principle}

In order to enhance the measurement of the kinematic fields and guide the code to converge to mechanically admissible solutions a mechanics-based regularization is applied [17]. An equivalent filter is derived to regularize the DL correction fields. The observed fluctuations (figure 5) are clearly not due to temperature (or DL) variations. A filter is designed and based upon a penalty given to the quadratic norm of the Laplacian of contrast and brightness fields. A functional (to be minimized), which is based upon the finite-element discretization of the Laplacian is introduced as

$$
\Phi_{D L}^{2}=\{\theta\}^{T}\left[L_{D L}\right]\{\theta\}=\{\theta\}^{T}\left[K_{D L}\right]^{T}[D]\left[K_{D L}\right]\{\theta\}
$$

where $\{\boldsymbol{\theta}\}$ is a vector gathering the nodal values of scalar fields $a$ and $b$. Note that $\left[\boldsymbol{K}_{\boldsymbol{D} \boldsymbol{L}}\right]$ is a matrix that naturally arises when solving a homogeneous heat conduction problem in steady state conditions and $[\boldsymbol{D}]$ is a diagonal matrix valued 1 for inner nodes and 0 for boundary ones. The recourse to such a regularization is to dampen out small fluctuations. For displacements, a similar strategy leads to a functional based on the equilibrium gap [17] 


$$
\Phi_{m}^{2}=\{\boldsymbol{u}\}^{T}\left[\boldsymbol{L}_{\boldsymbol{m}}\right]\{\boldsymbol{u}\}=\{\boldsymbol{u}\}^{T}\left[\boldsymbol{K}_{\boldsymbol{m}}\right]^{T}[\boldsymbol{D}]\left[\boldsymbol{K}_{\boldsymbol{m}}\right]\{\boldsymbol{u}\}
$$

where $[\boldsymbol{K}]$ is the stiffness matrix, $\{\boldsymbol{u}\}$ is the nodal displacement vector and $[\boldsymbol{D}]$ is the diagonal matrix valued 1 only for degrees of freedom of inner nodes, and 0 elsewhere. A block matrix containing both contributions (thermal and mechanical) for both fields (displacement and DL) is assembled

$$
\left[\mathbf{L}_{U}\right]=\left[\begin{array}{cc}
{\left[\boldsymbol{L}_{D L}\right]} & {[\mathbf{0}]} \\
{[\mathbf{0}]} & {\left[\boldsymbol{L}_{m}\right]}
\end{array}\right]
$$

For nodes near the ROI edges a similar strategy as in mechanics-based regularization can be used (for more details see Appendix A of Ref. [17]). Along boundaries, it is proposed to penalize the second derivatives of the scalar $a$ or $b$ fields

$$
\Phi_{b D L}^{2}=\{\boldsymbol{\theta}\}^{T}\left[\boldsymbol{L}_{b D L}\right]\{\boldsymbol{\theta}\}=\{\boldsymbol{\theta}\}^{T}\left[\boldsymbol{K}_{b D L}\right]^{T}\left[\boldsymbol{K}_{b D L}\right]\{\boldsymbol{\theta}\}
$$

where $\left[K_{b t}\right]$ is the (scalar) "boundary regularization" matrix while for boundary displacements [17]

$$
\Phi_{b m}^{2}=\{\boldsymbol{u}\}^{T}\left[\boldsymbol{L}_{\boldsymbol{b m}}\right]\{\boldsymbol{u}\}=\{\boldsymbol{u}\}^{T}\left[\boldsymbol{K}_{\boldsymbol{b m}}\right]^{T}\left[\boldsymbol{K}_{\boldsymbol{b m}}\right]\{\boldsymbol{u}\}
$$

where $\left[K_{b}\right]$ is the operator acting on the edges nodes [17]. A block matrix containing both contributions (thermal and mechanical) for edge nodes is constructed

$$
\left[\mathbf{L}_{b U}\right]=\left[\begin{array}{cc}
{\left[\boldsymbol{L}_{b D L}\right]} & {[\mathbf{0}]} \\
{[\mathbf{0}]} & {\left[\boldsymbol{L}_{b m}\right]}
\end{array}\right]
$$

The global functional to be minimized to estimate brightness, contrast and 2D displacement fields reads

$$
\left(1+\omega_{D L}+\omega_{m}+\omega_{b D L}+\omega_{b m}\right) \Phi_{t}^{2}=\Phi_{c}^{2}+\omega_{D L} \widehat{\Phi}_{D L}^{2}+\omega_{m} \widehat{\Phi}_{m}^{2}+\omega_{b D L} \widehat{\Phi}_{b D L}^{2}+\omega_{b m} \widehat{\Phi}_{b m}^{2}
$$

where $\omega_{D L}, \omega_{m}, \omega_{b D L}, \omega_{b m}$ are weights for the inner and boundary nodes for both mechanical and temperature (DL) regularizations. The defined residuals (in equations (1), (11), (12), (14) and (15)) are normalized [17]

$$
\widehat{\Phi}_{c}^{2}=\frac{\Phi_{c}^{2}}{\{\boldsymbol{V}\}^{t}[\boldsymbol{M}]\{\boldsymbol{V}\}}, \widehat{\Phi}_{D L}^{2}=\frac{\Phi_{D L}^{2}}{\{\boldsymbol{V}\}^{t}\left[\boldsymbol{L}_{\boldsymbol{D L}}\right]\{\boldsymbol{V} \boldsymbol{V}}, \widehat{\Phi}_{m}^{2}=\frac{\Phi_{m}^{2}}{\{\boldsymbol{V}\}^{t}\left[\boldsymbol{L}_{\boldsymbol{m}}\right]\{\boldsymbol{V}\}}, \widehat{\Phi}_{b D L}^{2}=\frac{\Phi_{b D L}^{2}}{\{\boldsymbol{V}\}^{t}\left[\boldsymbol{L}_{\boldsymbol{b D} \boldsymbol{L}}\right]\{\boldsymbol{V} \boldsymbol{V}\}}, \widehat{\Phi}_{b m}^{2}=\frac{\Phi_{b m}^{2}}{\{\boldsymbol{V}\}^{t}\left[\boldsymbol{L}_{\boldsymbol{b m}}\right]\{\boldsymbol{V}\}}
$$

The vector $\{\boldsymbol{V}\}$ collects the nodal values of the $\mathrm{DL}$ or displacement values related to a normalizing plane wave $\boldsymbol{V}$. The normalizing vector field is defined by $\boldsymbol{V}=\boldsymbol{V}_{\mathbf{0}} \exp (i \boldsymbol{k} \cdot \boldsymbol{x})$ of amplitudes $\boldsymbol{V}_{\mathbf{0}}=\left\{a_{0}, b_{0}, v_{0}\right\}=\mathbf{1}$ and wave vector $\boldsymbol{k}$. The regularization kernels act as fourth-order low pass filter while the DIC kernel $\{\boldsymbol{V}\}^{t}[\boldsymbol{M}]\{\boldsymbol{V}\}$ is wavelength independent [17]. They are linked to the regularization length (selected by the user) that reads for inner nodes

$$
\omega_{D L}=\left(2 \pi|\boldsymbol{k}| l_{D L}\right)^{4} \text { and } \omega_{m}=\left(2 \pi|\boldsymbol{k}| l_{m}\right)^{4}
$$

and for the boundary nodes

$$
\omega_{b D L}=\left(2 \pi|\boldsymbol{k}| l_{b D L}\right)^{4} \text { and } \omega_{b m}=\left(2 \pi|\boldsymbol{k}| l_{b m}\right)^{4}
$$

The regularization lengths $l_{D L}$ and $l_{b D L}$ are for (bulk and boundary respectively) DL fields regularization while $l_{m}$ and $l_{b m}$ are for the displacement fields. There are expressed in pixel units.

\section{Results and discussions}

The regularization effects on the $\mathrm{DL}$ fields are shown in figure 6 . When compared with figure 5 , the fluctuations are filtered out with a regularization length of 80 pixels for the $D L$ fields. 
(a)

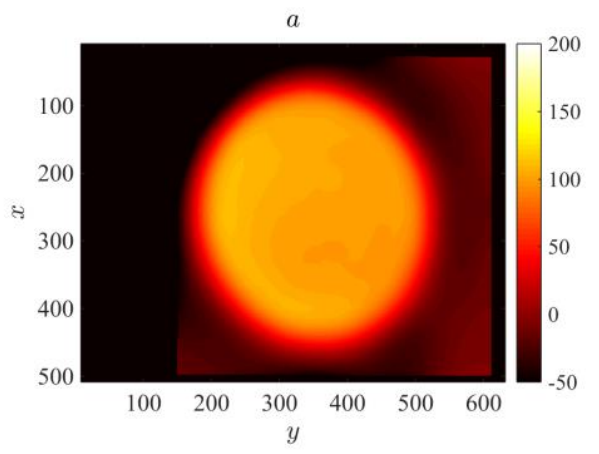

Fig 6: Regularized brightness

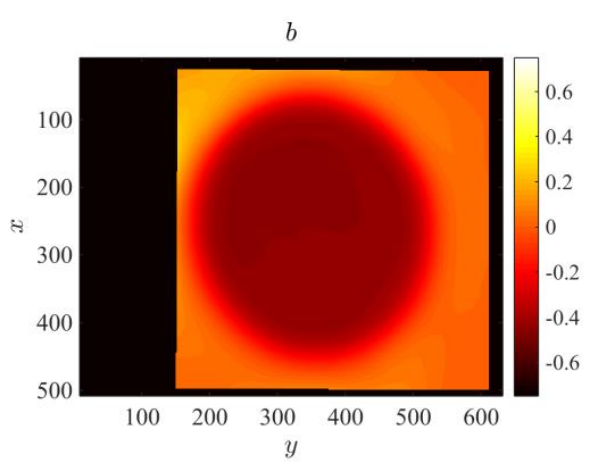

(b)

(a) and contrast (b) fields (to be compared with figure 5)

The root mean square (RMS) residual (i.e., the difference between the corrected deformed image and the reference image) is initially as high as $54 \%$ of the dynamic range. It is cut down to $0.93 \%$ after few iterations and reaches $0.58 \%$ at convergence. The residuals are mostly due to high local variations of DL and they vanish after a few iterations. The corrected deformed image in which the laser spot was present is shown in figure 7(a). As expected the residuals are higher near the laser spot edges (figure 7(b)). This may be due to two reasons. Either the element size or the regularization length is too large. The high gradients near the spot edges are penalized. If the mesh is fine enough these steep gradients may be freed.

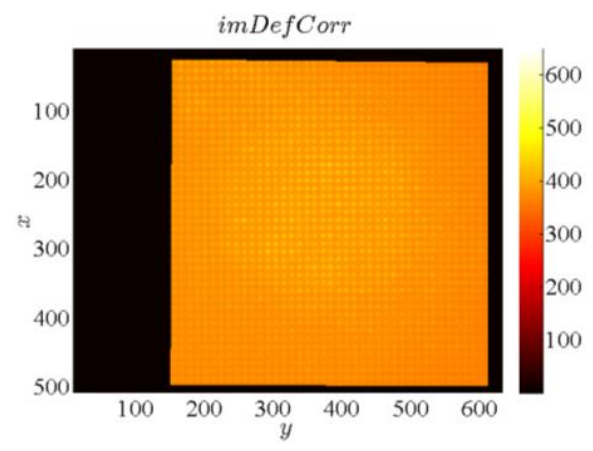

(a)

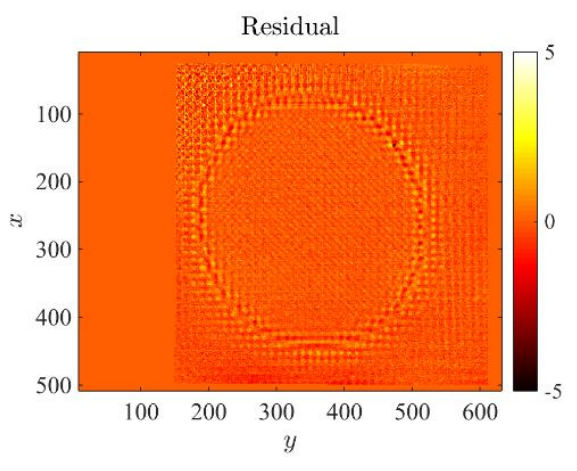

(b)

Fig 7: (a) Corrected deformed image and (b) residual map (RMS: $0.58 \%$ of the dynamic range) at convergence (to be compared with figure 2(a) and (c) respectively)

One possible strategy is to relax the regularization weight near the high DL gradient zones. The following writing is introduced for each elementary matrix

$$
\left[\tilde{\boldsymbol{L}}_{D L}^{e}\right]=(1-D)\left[\boldsymbol{L}_{D L}^{e}\right]
$$

where D acts as a "damage" parameter varying from 0 ("undamaged" state) to 1 (fully "damaged" state)

$$
D=1-\exp \left(-\left(\frac{\Delta a}{a_{\text {crit }}}\right)^{2}\right)
$$

where $\Delta$ is the Laplacian operator and the threshold $a_{\text {crit }}$ is selected as being equal to $0.8 \max (|\Delta a|)$. Only one "damage" field affecting the contrast or brightness is needed as they share the same sharp gradient zones. The results in terms of "damage" and brightness fields are shown in figure 8. 

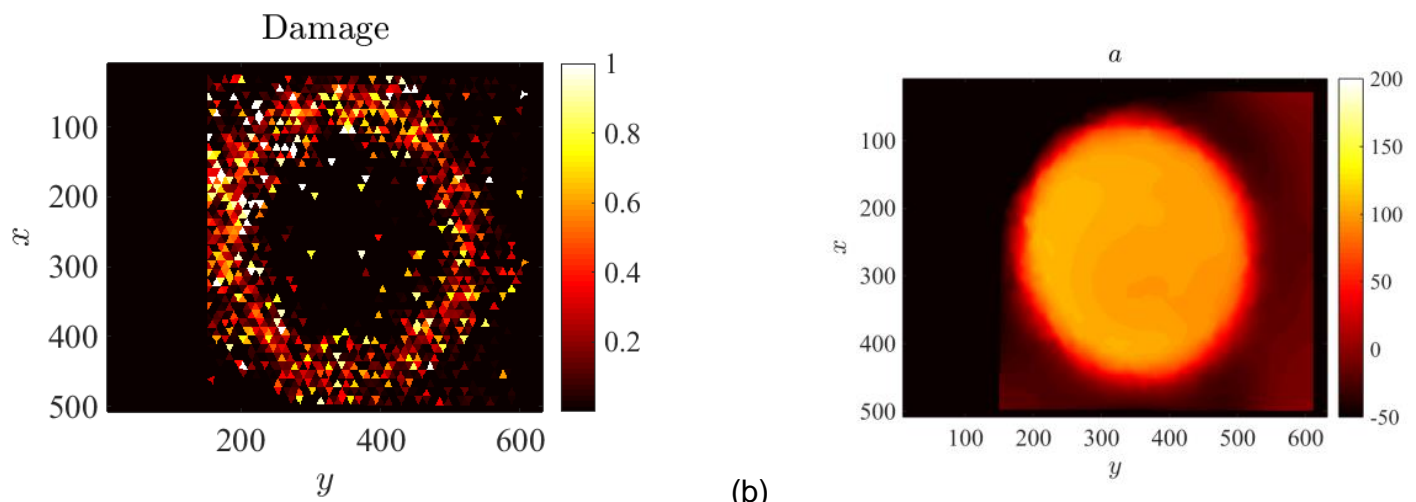

(a)

Fig 8: (a) Damage and (b) brightness fields with an adapted DL regularization with damage

It is observed that the affected elements are those near the edges as could be expected (figure 8(a)) since the regularization is lowered in those particular zones (figure $8(\mathrm{~b})$ ). The RMS residuals are reduced to $0.56 \%$ (against $0.58 \%$ before introducing the "damage" based regularization). The "crown" in figure 7 is still observed on the residual map (see figure 9) but with a smaller thickness, which was the aim of the proposed procedure.

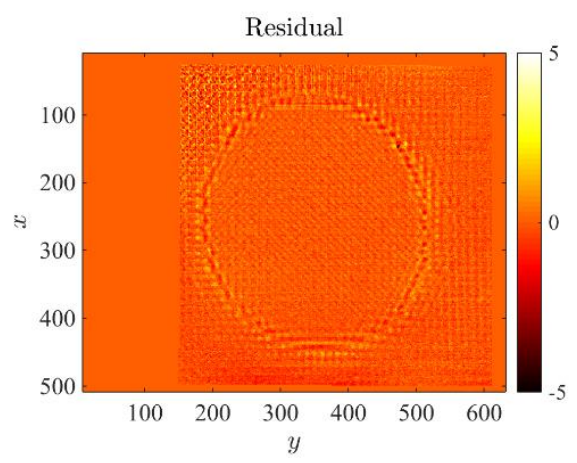

Fig 9: Residuals map (RMS average: $0.56 \%$ ) after readapting the DL regularization with the "damage" field at convergence (to be compared with $0.58 \%$ )

The displacement results are quite comparable to the measurements obtained with the synchronized visible light camera, which is not disturbed by the very high local digital level variations induced by heating. The displacement fields correspond to a biaxial loading state as expected from numerical simulations [14].

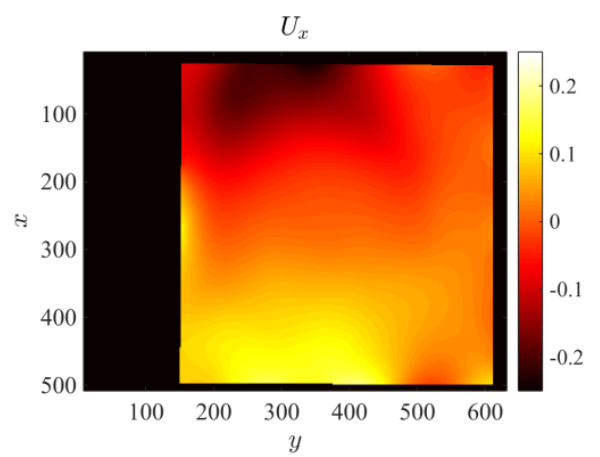

(a)

Fig 10: Displacement fields at the end of a thermal shock expressed in pixel ( 1 pixel $=15 \mu \mathrm{m})$.

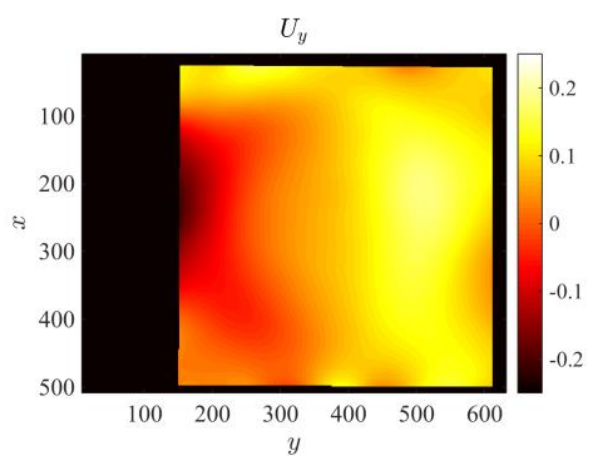

(a) Vertical $U_{x}$ and (b) horizontal $U_{y}$ components

\section{Conclusions and prospects}

A DIC algorithm is proposed to measure 2D displacements while large and abrupt digital level variations occur between the registered infrared images. The DL variation is accounted for by introducing spatially varying brightness and 


\subsection{1/qirt.2016.019}

contrast corrections in the DIC formalism. Then by decomposing the unknown fields onto an FE basis, IRIC could be successfully performed. The results provide a good estimation of the displacement fields with element size of 12 pixels but the DL fields are "noisy" as they are limited by the mesh size. When a finer mesh is used, the ill-posedness of the DIC problem calls for a regularization strategy to be applied to the displacement and the DL correction fields. This regularization provides satisfactory results. To progress further, it is necessary to relax the regularization in zones where sharp gradients are observed using DL gradient-dependent "damage" parameter. This strategy provides displacement results that are quite comparable to the measurements obtained with the synchronized visible light camera but are not perturbed by the very high local digital level variations induced by heating. In the future, the code will be compared against numerical test cases in order to estimate its resolution and noise robustness [20].

\section{REFERENCES}

[1] A. Chrysochoos and G. Martin, "Tensile test microcalorimetry for thermomechanical behaviour law analysis," Mater. Sci. Eng. A, vol. 108, pp. 25-32, Feb. 1989.

[2] D. Favier, H. Louche, P. Schlosser, L. Orgéas, P. Vacher, and L. Debove, "Homogeneous and heterogeneous deformation mechanisms in an austenitic polycrystalline Ti-50.8 at.\% Ni thin tube under tension. Investigation via temperature and strain fields measurements," Acta Mater., vol. 55, no. 16, pp. 5310-5322, Sep. 2007.

[3] P. Schlosser, H. Louche, D. Favier, and L. Orgéas, "Image Processing to Estimate the Heat Sources Related to Phase Transformations during Tensile Tests of NiTi Tubes," Strain, vol. 43, no. 3, pp. 260-271, Aug. 2007.

[4] A. Chrysochoos, B. Berthel, F. Latourte, A. Galtier, S. Pagano, and B. Wattrisse, "Local energy analysis of high-cycle fatigue using digital image correlation and infrared thermography," J. Strain Anal. Eng. Des., vol. 43, no. 6, pp. 411422, Jun. 2008.

[5] A. Chrysochoos, "Thermomechanical Analysis of the Cyclic Behavior of Materials," IUTAM Symp. Full-Field Meas. Identif. Solid Mech., vol. 4, pp. 15-26, 2012.

[6] L. Bodelot, L. Sabatier, E. Charkaluk, and P. Dufrénoy, "Experimental setup for fully coupled kinematic and thermal measurements at the microstructure scale of an AISI 316L steel," Mater. Sci. Eng. A, vol. 501, no. 1-2, pp. 52-60, Feb. 2009.

[7] R. Seghir, E. Charkaluk, P. Dufrénoy, and L. Bodelot, "Thermomechanical couplings in crystalline plasticity under fatigue loading," Fatigue 2010, vol. 2, no. 1, pp. 1155-1164, Apr. 2010.

[8] L. Bodelot, E. Charkaluk, L. Sabatier, and P. Dufrénoy, "Experimental study of heterogeneities in strain and temperature fields at the microstructural level of polycrystalline metals through fully-coupled full-field measurements by Digital Image Correlation and Infrared Thermography," Mech. Mater., vol. 43, no. 11, pp. 654-670, Nov. 2011.

[9] R. Seghir, L. Bodelot, E. Charkaluk, and P. Dufrénoy, "Numerical and experimental estimation of thermomechanical fields heterogeneity at the grain scale of $316 \mathrm{~L}$ stainless steel," Comput. Mater. Sci., vol. 53, no. 1, pp. 464-473, Feb. 2012.

[10] S. Utz, E. Soppa, K. Christopher, X. Schuler, and H. Silcher, "Thermal and mechanical fatigue loading - Mechanisms of crack initiation and crack growth," presented at the Proceedings of the ASME 2014 Pressure Vessels \& Piping Conference, PVP2014, Anaheim, California, USA, 2014, p. 10

[11] T. Pottier, M.-P. Moutrille, J.-B. Le Cam, X. Balandraud, and M. Grédiac, "Study on the Use of Motion Compensation Techniques to Determine Heat Sources. Application to Large Deformations on Cracked Rubber Specimens," Exp. Mech., vol. 49, no. 4, pp. 561-574, Aug. 2009.

[12] A. Maynadier, M. Poncelet, K. Lavernhe-Taillard, and S. Roux, "One-shot Measurement of Thermal and Kinematic Fields: InfraRed Image Correlation (IRIC)," Exp. Mech., vol. 52, no. 3, pp. 241-255, Mar. 2012.

[13] C. Esnoul, L. Vincent, M. Poncelet, F. Hild, and S. Roux, "On the use of thermal and kinematic fields to identify strain amplitudes in cyclic laser pulses on AISI 304L strainless steel," presented at the Photomechanics conference, Montpellier, 2013.

[14] A. Charbal, L. Vincent, F. Hild, M. Poncelet, J.-E. Dufour, S. Roux, and D. Farcage, "Characterization of temperature and strain fields during cyclic laser shocks," Quant. InfraRed Thermogr. J., vol. 13, no. 1, pp. 1-18, 2016.

[15] F. Hild and S. Roux, "Digital Image Correlation," in Optical Methods for Solid Mechanics : A Full-Field Approach, WileyVCH, Berlin (Germany). P. Rastogi and Editor E. Hack (Edts.), 2012.

[16] G. Besnard, F. Hild, and S. Roux, "Finite-Element' Displacement Fields Analysis from Digital Images: Application to Portevin-Le Châtelier Bands," Exp. Mech., vol. 46, no. 6, pp. 789-803, 2006.

[17] Z. Tomicevic, F. Hild, and S. Roux, "Mechanics-aided digital image correlation," J. Strain Anal. Eng. Des., vol. 48, no. 5, pp. 330-343, 2013.

[18] L. Vincent, M. Poncelet, S. Roux, F. Hild, and D. Farcage, "Experimental Facility for High Cycle Thermal Fatigue Tests Using Laser Shocks," Fatigue Des. 2013 Int. Conf. Proc., vol. 66, pp. 669-675, 2013.

[19] F. Hild and S. Roux, "Comparison of Local and Global Approaches to Digital Image Correlation," Exp. Mech., vol. 52, no. 9, pp. 1503-1519, Nov. 2012.

[20] A. Charbal, J.-E. Dufour, A. Guery, F. Hild, S. Roux, L. Vincent, and M. Poncelet, "Integrated Digital Image Correlation considering gray level and blur variations: Application to distortion measurements of IR camera," Opt. Lasers Eng., vol. 78, pp. 75-85, Mar. 2016. 